\title{
Contrôle de l'activité lymphocytaire par les granules de stress
}

\section{Nouvelles cibles pour l'immunothérapie?}

Don-Marc Franchini ${ }^{1,2}$, Olivia Lanvin ${ }^{1,2}$, Nicolas Curdy ${ }^{1,2}$, Jean-Jacques Fourniél,2

> Les cellules eucaryotes perçoivent comme des stress une multitude de situations endogènes ou environnementales telles que la réplication de I'ADN, un choc thermique, une infection virale, ou le voisinage de cellules transformées. Elles ont donc développé des processus génériques de réponse au stress qui contribuent à l'homéostasie. Chez l'homme, un certain nombre de maladies neurodégénératives et de cancers s'accompagnent d'anomalies de réponse au stress. Outre les mécanismes de réparation de l'ADN ou de contrôle du cycle cellulaire, les cellules peuvent contrôler le métabolisme de leurs ARN messagers (ARNm) afin de modifier rapidement l'expression de leurs gènes. Ce contrôle peut s'effectuer au niveau transcriptionnel, en modulant le niveau de production des ARNm dans le noyau de la cellule, ou à un stade posttranscriptionnel, en mobilisant les ARNm déjà produits au sein de structures cytoplasmiques comme les processing bodies [1] $(\rightarrow)$ ou les $(\rightarrow)$ Voir la Nouvelle de M. Courel et al., $\mathrm{m} / \mathrm{s} \mathrm{n}^{\circ} 4$, avril 2018 , page 306

$(\rightarrow)$ Voir la Nouvelle de J. Nikolic et $D$. Blondel, $m / s n^{\circ} 11$, novembre 2017, page 921 granules de stress [2] $(\rightarrow)$.

Ces structures présentent la particularité d'être dépourvues de membrane, et se forment par l'agrégation progressive d'ARNm et de protéines pour constituer des granules [3]. Les ARNm localisés dans ces granules sont maintenus dans un état non-traduit, et peuvent y être dégradés [4]. Cependant, la nature transitoire, dynamique et réversible de ces granules permet leur désassemblage une fois le stress dissipé, ce qui facilite la reprise de la traduction des ARNm qu'ils contiennent [5].

La réponse au stress est bien étudiée dans des types cellulaires neuronaux ou cancéreux exposés à un stress osmotique, thermique, ou chimique. Les cellules du système immunitaire, dont les lymphocytes, nécessitent quant à elles une stimulation pour être pleinement fonctionnelles. Cette stimulation provient de la reconnaissance d'un antigène par les récepteurs qu'elles expriment ( $T$-cell receptor $[\mathrm{TCR}]$ pour les lymphocytes $T, B$-cell receptor $[B C R]$, constitué d'une immunoglobuline de surface et de chaînes associées, pour les lymphocytes $B$ ), et induit une reprogrammation transcriptionnelle des lymphocytes pour déclencher leurs programmes de prolifération, de différenciation et de fonctions effectrices. Pour assurer le bon déroulement de chacun de ces processus biologiques, l'expression des protéines effectrices de ces différents programmes est donc minutieusement orchestrée. Loin d'être anodine, la stimulation des lymphocytes induit l'expression de facteurs spécifiques des granules de stress. Bien que le rôle de ces granules ne soit pas encore complétement élucidé, il semble qu'ils contrôlent l'expression de facteurs cruciaux pour la réponse immunitaire.
${ }^{1}$ Centre de Recherches en Cancérologie de Toulouse, Inserm UMR1037 ; Université Toulouse III Paul Sabatier ; CNRS ERL5294, 2, avenue Hubert Curien, 31037 Toulouse, France.

${ }^{2}$ Institut universitaire du cancer de Toulouseoncopole, 1, avenue Irène Joliot-Curie, 31059 Toulouse, France.

don-marc.franchini@inserm.fr

\section{Granules de stress et diversification} des immunoglobulines

Dans les centres germinatifs, la stimulation des lymphocytes B induit l'expression de la protéine AID (activationinduced cytidine deaminase) qui est à l'origine des réactions de commutation isotypique et d'hypermutation somatique pour augmenter l'affinité et parfois améliorer la spécificité des immunoglobulines envers l'antigène [6]. Les voies de réponse aux dommages subis par I'ADN jouent un rôle prépondérant pour le bon déroulement de ces réactions, tout en assurant le maintien de l'intégrité du génome. Pour cela, l'ARNm codant la protéine p53, un facteur clé de la réponse aux lésions de l'ADN, se retrouve localisé dans les granules de stress générés par la stimulation des lymphocytes B [7]. Cependant, ce n'est qu'une fois que les cassures de I'ADN se sont produites que, sous l'effet de l'activation de la kinase ATM (ataxia telangiectasia mutated), I'ARNm codant p53 se dissocie des granules de stress. Cette délocalisation permet ainsi sa traduction, et le déclenchement des voies de réparation des cassures de l'ADN. Curieusement, dans les lymphocytes B, l'inhibition par la rapamycine de mTOR (mechanistic target of rapamycin), un facteur associé à la prolifération et à la survie cellulaires, diminue la quantité de protéine AID mais pas celle de son transcrit [8]. Ce contrôle posttranscriptionnel dépend de la protéine $4 \varepsilon-B P l$ (eukaryotic translation initia- 


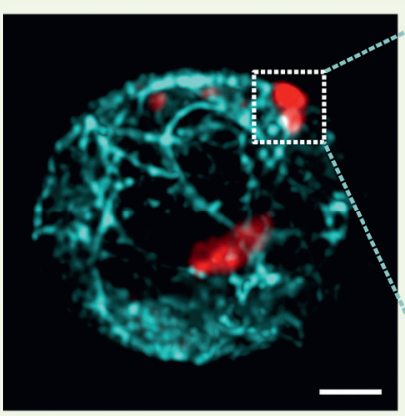

Complexe

ribonucléoprotéique

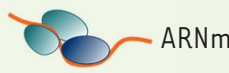

Traduction
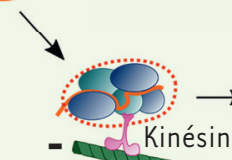

Granules de stress

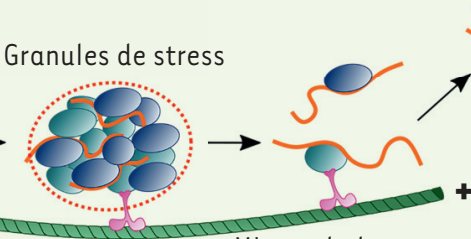

Microtubule

Figure 1. Les microtubules et les granules de stress régulent l'expression des points de contrôle immunitaire dans les lymphocytes T activés. Image d'un lymphocyte T activé réalisée en microscopie confocale à haute résolution. Les microtubules sont visualisés par un marquage de la tubuline (en cyan) et les granules de stress par un marquage de la protéine G3BPI (Ras-GTPase-activating protein SH3-domain-binding protein 1) (en rouge). Barre d'échelle : $2 \mu \mathrm{m}$. Le schéma résume les mécanismes du contrôle post-transcriptionnel des ARNm des récepteurs inhibiteurs de la réponse immunitaire (en orange) par les granules de stress. Ces ARNm forment des complexes ribonucléoprotéiques qui s'agrègent progressivement en granules de stress. Les microtubules, via les kinésines, contrôlent la dynamique de ces granules de stress et permettent le transport des ARNm jusqu'aux ribosomes pour leur traduction.

tion factor $4 \varepsilon$-binding protein 1) qui contribue notamment à la formation des granules de stress. Bien que leur implication exacte reste à définir, ces observations suggèrent que les granules de stress contrôlent la différenciation des lymphocytes $B$, notamment par un contrôle temporel de la commutation isotypique.

\section{Granules de stress et points de contrôle immunitaire}

À côté de la régulation de la production des immunoglobulines par les lymphocytes $B$, le contrôle de la réponse immunitaire repose également sur des points de contrôle immunitaire. Ces récepteurs, qui peuvent être stimulateurs ou inhibiteurs, modulent l'activation des lymphocytes $T$. Les récepteurs inhibiteurs, tels que PD-1 (programmed cell death-1) ou CTLA-4 (cytotoxic T-lymphocyte-associated antigen 4), sont exprimés à la surface des lymphocytes activés et jouent un rôle prépondérant dans la réponse immunitaire, notamment dans la tolérance. Chez la plupart des patients souffrant de cancers, les cellules tumorales surexpriment les ligands de ces récepteurs, et tirent ainsi profit de l'engagement de ces freins immunitaires pour inhiber les fonctions lymphocytaires, empêchant ainsi leur élimination. La découverte de ces points de contrôle et de leur rôle en cancérologie a permis une véritable révolution thérapeutique avec la production d'anticorps monoclonaux capables de cibler ces récepteurs et ainsi de désinhiber et d'amplifier la réponse des lymphocytes. Pour de nombreux cancers, l'utilisation de ces anticorps au cours d'essais cliniques a produit des résultats remarquables, et l'enjeu actuel est d'en améliorer encore l'efficacité $[9](\rightarrow)$.

Toutefois, malgré

$(\rightarrow)$ Voir l'Éditorial de S. Champiat et J.C. Soria, $m / s n^{\circ} 6-7$, juin-juillet 2017, page 563

l'importance clinique de l'expression de ces récepteurs par les lymphocytes infiltrant les tumeurs, les mécanismes du contrôle de cette expression restent mal connus.

Nous avons récemment découvert un mécanisme de contrôle post-transcriptionnel, assez semblable à celui impliqué dans le contrôle de l'expression des récepteurs synaptiques dans les neurones (Figure 1) [10]. Nous avons en effet observé que, à la suite de leur stimulation, des facteurs spécifiques des granules de stress tels que G3BPl (RasGTPase-activating protein SH3-domainbinding protein 1) étaient exprimés par les lymphocytes T humains, et qu'ils s'as- sociaient aux ARNm codant ces points de contrôle immunitaire. Ces complexes ribonucléoprotéiques sont acheminés par la kinésine 1 , un moteur moléculaire composé des protéines KIF5B (kinesin family member $5 B$ ) et $\mathrm{KLCl}$ (kinesin light chain 1), le long des microtubules pour former des granules de stress. Sans ce moteur moléculaire ou sans les microtubules, le transport et donc la maturation des granules de stress restent bloqués dans un état qui ne permet pas leur désassemblage. Cela empêche alors le transfert des ARNm aux polysomes pour leur traduction en protéines. Étonnamment, il est apparu que les granules de stress exercent un contrôle sur la plupart des récepteurs inhibiteurs de la réponse immunitaire (PD-1, CTLA-4, LAG3 [lymphocyte activation gene 3], TIM3 [T cell immunoglobulin mucin 3]), mais n'ont aucune action sur les récepteurs stimulateurs (GITR [glucocorticoid-induced tumor necrosis factor (TNF)-related protein ], 0X40, 4-1BB). Une caractéristique essentielle des ARNm dont la traduction en protéines est contrôlée par les granules de stress semble être la longueur de leur partie non-codante 3'UTR (untranslated region). Pour les ARNm codant les récepteurs inhibiteurs des lymphocytes, cette longueur est similaire à celle des 
ARNm neuronaux transportés le long des microtubules vers leur site de traduction sous la synapse neuronale. Dans les lymphocytes, ce mécanisme de régulation du transport des ARNm est retrouvé dans l'ensemble du lignage $\mathrm{T} \alpha \beta\left(\mathrm{CD} 8^{+}\right.$et $\left.\mathrm{CD} 4^{+}\right)$ et $\mathrm{T} \gamma \delta$.

Parmi les agents chimiothérapeutiques, les molécules ciblant les microtubules sont utilisées pour leur capacité à inhiber la prolifération cellulaire, ce qui permet de diminuer la croissance de certaines tumeurs. L'analyse de l'expression des points de contrôle immunitaire par les lymphocytes $T$ intra-tumoraux chez des patients atteints d'un lymphome de Hodgkin ayant reçu un traitement contenant un agent ciblant les microtubules a montré qu'elle était significativement réduite. Une des principales caractéristiques cliniques des anticorps ciblant ces récepteurs est de fréquemment provoquer des effets secondaires auto-immuns. Nous avons interrogé la base de données établie par l'Organisation mondiale de la santé (Vigibase), qui recense l'ensemble des effets secondaires pour tous types de médicaments dans tous types d'indications. Cette enquête rétrospective à très grande échelle (200000 patients) a révélé que les médicaments inhibiteurs des microtubules présentent aussi un risque accru de réaction auto-immune. Au-delà de leur activité antimitotique exploitée en cancérologie, ces composés peuvent donc aussi influencer l'im- munité. En modulant l'expression des récepteurs inhibiteurs à la surface des cellules immunitaires, les médicaments inhibiteurs de microtubules pourraient donc contribuer à de nouvelles stratégies thérapeutiques anticancéreuses.

Comme pour le réseau de microtubules, l'assemblage des granules de stress peut aussi être bloqué pharmacologiquement. Ainsi, le ciblage des granules de stress ouvre la perspective de moduler l'expression des récepteurs inhibiteurs des lymphocytes $T$ dans le cadre de nouvelles stratégies immunothérapeutiques.

\section{Conclusions}

Les granules de stress jouent donc un rôle majeur et inattendu dans le contrôle post-transcriptionnel des ARNm dans les cellules du système immunitaire. Audelà de leur fonction protectrice visà-vis d'un stress, ces granules exercent également un contrôle temporel de la traduction des ARNm, permettant d'ajuster le déroulement de processus biologiques spécifiques. Ils pourraient ainsi constituer une nouvelle classe de cibles immunothérapeutiques. $\diamond$ Control of lymphocyte activity by stress granules. New targets for immunotherapy?

\section{REMERCIEMENTS}

Ce travail a été soutenu par la Fondation ARC pour la recherche sur le cancer (équipe labélisée), la Ligue contre le cancer, le Cancéropôle Grand SudOuest et le Labex TouCan.

\section{LIENS D'INTÉRÊT}

D.M. Franchini, 0. Lanvin, et J.-J. Fournié sont auteurs des brevets Inserm EP17305514 et EP18306286.8 issus de cette étude.

J.-J. Fournié est membre du conseil consultatif ou scientifique pour les laboratoires ImCheck Therapeutics, American Gene Technology et Boehringer-Ingelheim.

$N$. Curdy déclare n'avoir aucun lien d'intérêt concernant les données publiées dans cet article.

\section{RÉFÉRENCES}

1. Courel M, Bénard M, Ernoult-Lange M, et al. Les $P$-bodies: des gouttelettes microscopiques pour stocker les messagers de protéines régulatrices. Med Sci (Paris) 2018; $34: 306-8$.

2. Nikolic J, Blondel D. Le virus de la rage induit la formation de granules de stress en contact étroit avec les usines virales. Med Sci (Paris) 2017 ; 33 : 921-3.

3. Van Treeck B, Parker R. Emerging roles for intermolecular RNA-RNA interactions in RNP assemblies. Cell $2018 ; 174: 791-802$.

4. Ivanov P, Kedersha N, Anderson P. Stress granules and processing bodies in translational control. Cold Spring Harbor Perspect Biol 2019 ;11. pii: a032813.

5. Protter DSW, Parker R. Principles and properties of stress granules. Trends Cell Biol $2016 ; 26$ : 668-79.

6. Franchini DM, Petersen-Mahrt SK. AID and APOBEC deaminases: balancing DNA damage in epigenetics and immunity. Epigenomics $2014 ; 6:$ 427-43.

7. Díaz-Muñoz MD, Kiselev Vy, Le Novère N, et al. Tialdependent regulation of mRNA subcellular location and translation controls $p 53$ expression in B cells. Nat Commun $2017 ; 8: 530$.

8. Chiu H, Jackson LV, Oh KI, et al. The mTORCl/4E-BP/ elF4E axis promotes antibody class switching in $B$ lymphocytes. J Immunol 2019 ; 202 : 579-90.

9. Champiat S, Soria JC. Nouvelles pratiques en immunooncologie : une révolution et un immense défi scientifique et médical. Med/Sci (Paris) 2017 ; 33 : 563-4.

10. Franchini D-M, Lanvin 0, Tosolini M, et al. Microtubule-driven stress granule dynamics regulate inhibitory immune checkpoint expression in T cells. Cell Rep 2019 ; 26 : 94-107.e7.

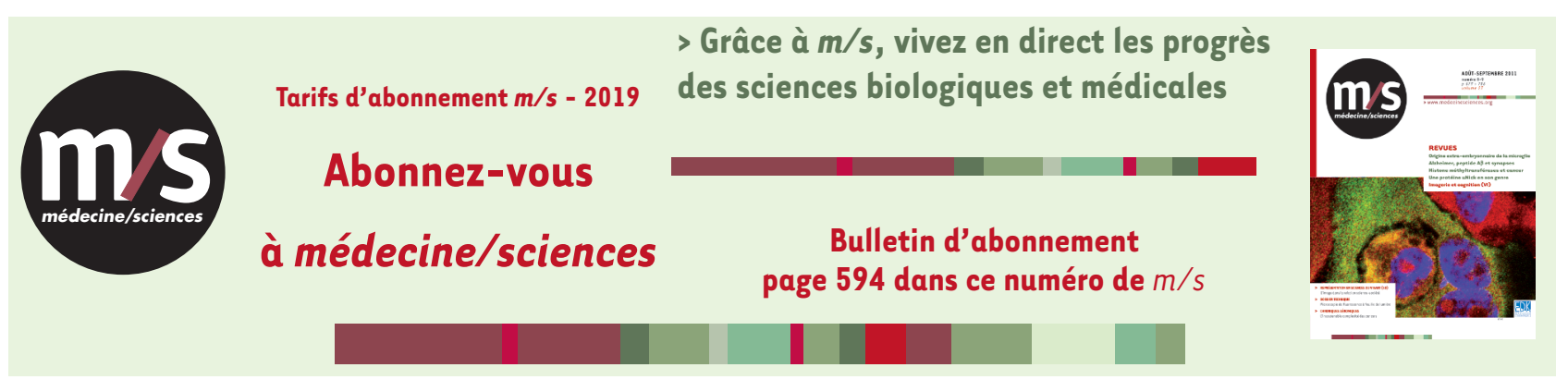

